\title{
Transfer of Parahydrogen Induced Polarization in Scalar Coupled Systems at Variable Magnetic Field
}

\author{
By Alexey S. Kiryutin ${ }^{1,2}$, Konstantin L. Ivanov ${ }^{2,3}$, Alexandra V. Yurkovskaya ${ }^{2,3}$, \\ Robert Kaptein $^{3,4}$, and Hans-Martin Vieth ${ }^{1, *}$ \\ ${ }^{1}$ Institut für Experimentalphysik, Freie Universität Berlin, Arnimallee 14, 14195 Berlin, Germany \\ 2 International Tomography Center, Institutskaya 3a, Novosibirsk, 630090, Russia \\ ${ }^{3}$ Novosibirsk State University, Pirogova 2, Novosibirsk, 630090, Russia \\ ${ }^{4}$ Bijvoet Center for Biomolecular Research, Utrecht University, Padualaan 8, NL-3584, The Netherlands
}

\section{Dedicated to Professor Hans Wolfgang Spiess on the occasion of his $70^{\text {th }}$ birthday}

(Received June 26, 2012; accepted in revised form August 27, 2012)

(Published online October 29, 2012)

\section{Spin Hyperpolarization / Polarization Transfer / Para-Hydrogen / PHIP / Magnetic Field Dependence}

Para-Hydrogen Induced Polarization (PHIP) experiments were performed in coupled multispin systems at variable magnetic fields. We studied the magnetic field dependence of PHIP in styrene, which is the product of hydrogenation of phenylacetylene. At low magnetic fields where the spins are coupled strongly by scalar interaction efficient polarization transfer among the interacting protons takes place. The experimentally observed spectra are in good agreement with the simulation, which takes into account eight coupled spins. We also demonstrate effects of nuclear spin level anti-crossings on the PHIP pattern. It is shown that rapid passage through the level anti-crossing enables highly efficient polarization transfer between specific spin orders. In addition, we studied PHIP transfer to ${ }^{13} \mathrm{C}$ and ${ }^{19} \mathrm{~F}$ hetero-nuclei. It is shown that hetero-nuclei can be efficiently polarized in a wide field range; in particular, for polarizing them it is not necessary to go to ultra-low fields, which provide their strong coupling to protons. The resulting polarization is of the multiplet type and gives strong enhancements of the individual NMR lines. In general, variation of the magnetic field gives the opportunity for manipulating PHIP patterns and transferring polarization to target spins of choice.

\section{Introduction}

Para-Hydrogen Induced Polarization (PHIP) [1,2] is a particularly powerful method for enhancing the NMR signals by several orders of magnitude [3]. PHIP can be formed by catalytic attachment of a para-dihydrogen molecule, i.e., a $\mathrm{H}_{2}$ molecule in its singlet nuclear spin state, to molecules with a double or triple $\mathrm{C}-\mathrm{C}$ bond. In its turn, the para-

* Corresponding author. E-mail: hans-martin.vieth@physik.fu-berlin.de 
component of dihydrogen gas can be easily enriched at low temperature. An advantage of the technique is that it is relatively simple and general enabling to achieve spin polarization, which is 3-4 orders of magnitude higher than the Boltzmann polarization at room temperature, resulting in anomalous NMR spectra of the hydrogenation products. A prerequisite for PHIP is that the two hydrogen atoms become non-equivalent in the reaction products. This enables transfer of the initial singlet spin order, which is NMR silent, into observable NMR signals. Although the integral over the whole PHIP spectrum is zero the individual lines are enhanced enormously. PHIP can also be formed in reversible reactions of substrates with para-hydrogen that do not require hydrogenation [4].

PHIP experiments are usually performed according to the two conventional PHIP protocols termed ALTADENA (adiabatic longitudinal transport after dissociation engenders nuclear alignment) [5] and PASADENA (parahydrogen and synthesis allow dramatically enhanced nuclear alignment) [6]. In the ALTADENA case the hydrogenation reaction is performed at low magnetic field (presumably, the Earth's field) and the polarized reaction products are transferred adiabatically to the high field of the NMR spectrometer where the NMR spectrum is taken. In the PASADENA experiment the hydrogenation is performed at the high field of the NMR spectrometer, hence, no additional sample transfer step is required. In both cases the PHIP spectra exhibit large non-thermal polarization although the spectral pattern is different: in the PASADENA case only multiplet polarization of spins is formed whereas in the ALTADENA case there is also net polarization of spins seen.

In, general, variation of the external magnetic field gives a way to manipulate PHIP spectral patterns. This is because by going through the field one can choose the regime of coupling between the spins. Two spins are considered weakly coupled once their spin-spin interaction, $J$, is much smaller than the difference in their Zeeman interaction with the field, $\delta v$. In the opposite case the spins are coupled strongly. Since $\delta v$ is directly proportional to the magnetic field strength the weak coupling and strong coupling regimes correspond to high and low magnetic fields, respectively. The eigen-states of the weakly and strongly coupled system differ: for the simplest weakly-coupled twospin $1 / 2$ system the eigen-states are the Zeeman product states whereas for the strongly coupled system these are the collective singlet and three triplet states. As a consequence, different PHIP patterns are observed when the system is polarized at low or high magnetic fields. Strong coupling of spins can also lead to efficient polarization transfer between them because they have collective states and therefore get polarized simultaneously [7-10]. Such polarization transfer effects are of a coherent nature and depend also on the magnetic field [9-12].

Thus, varying the magnetic field allows one not only to change the polarization pattern of a pair of spins but also to transfer PHIP to other nuclei. Nonetheless, this attractive possibility has not been fully exploited so far. There are only a few examples [13-17] of PHIP experiments with polarization preparation done at variable magnetic fields. To bridge this gap we performed PHIP at different magnetic fields in a range of 0 to 7 Tesla. We have considered polarization patterns in a system of three coupled protons and studied polarization transfer to spin $1 / 2$ hetero-nuclei, namely, to ${ }^{13} \mathrm{C}$ and ${ }^{19} \mathrm{~F}$. In addition, we investigated the possibility of manipulating PHIP by fast switching of the external magnetic field. Experiments were per- 
formed by using a fast field-cycling device [18,19] that enabled (i) high-resolution NMR detection and (ii) field variation in the range between 0 and $7 \mathrm{~T}$ with a precisely known time profile of switching from the polarization to the detection field. Our experimental setup also allowed us to conduct more complicated experiments with two field jumps, which is important for manipulating the initial spin order and transferring PHIP to other nuclei. We also modified the NMR sample tubes and introduced a device for performing the hydrogenation reaction at arbitrary field to measure the PHIP field dependence in a precise and reproducible way. The experimental results for PHIP on protons were supported by theoretical simulations performed in the framework of the approach elaborated recently [10] that has proven its high accuracy [13].

\section{Experimental}

\subsection{Chemical compounds}

Chemicals used in this study are as follows: phenylacetylene-98\%, 3,4-difluorophenylacetylene $90 \%$, ethyl phenylpropiolate $98 \%$ and the precatalyst of the hydrogenation reaction [1,4-bis(diphenylphosphino)butane] (1,5-cyclooctadiene)rhodium(I) tetrafluoroborate $98 \%$. They were obtained from Sigma-Aldrich and used without any further purification. 4-fluorophenylacetylene 99\% was bought from ABCR GmbH, deuterated solvents acetone- $\mathrm{d}_{6} 99.8 \%$ and methanol- $\mathrm{d}_{4} 99.8 \%$ were purchased from Deutero $\mathrm{GmbH}$. Para-hydrogen was prepared in a brass U-tube under pressure and used directly in the same way as previously [13]. The vessel contained charcoal as an ortho-para conversion catalyst; its surface was activated under vacuum for two hours prior to the experiments. The part of the U-tube containing charcoal was submerged in liquid nitrogen; subsequently the $\mathrm{H}_{2}$ gas was added at a pressure of 2 bars. Under these conditions $(77 \mathrm{~K})$, a mixture of $51 \%$ para-hydrogen and $49 \%$ ortho-hydrogen is generated after approximately $2 \mathrm{~h}$. Such a content of para-hydrogen far exceeds that found at thermal equilibrium at ambient conditions and the resulting polarization can thus be regarded as hyperpolarization.

The structures of the compounds used and catalytic chemical reactions that were utilized to produce PHIP by hydrogenation are shown in Chart 1. Phenylacetylene and its F-containing derivatives can be hydrogenated to yield styrene and its fluorinated analogues. In the presence of the catalyst these reactions are very efficient [20] and run under atmospheric pressure. Ethyl phenylpropiolate can be efficiently hydrogenated catalytically [21] and gives huge proton PHIP signals of ethyl-cinnamate.

Solutions of phenylacetylene $(200 \mathrm{mmol} / \mathrm{l})$ and 4-fluorophenylacetylene $(200 \mathrm{mmol} / \mathrm{l})$ were prepared in acetone- $\mathrm{d}_{6}$, concentration of the precatalyst was $2 \mathrm{mmol} / \mathrm{l}$. Solutions of ethyl phenylpropiolate $(500 \mathrm{mmol} / \mathrm{l})$ were prepared in methanol- $\mathrm{d}_{4}$ with the same concentration of the precatalyst. Each solution was prepared immediately prior to the experiment because of the degradation of the catalyst in the solution. For each measurement in a different field a new sample was prepared with exactly the same concentrations. The solutions were bubbled with nitrogen for $15 \mathrm{~min}$ to remove dissolved oxygen. 


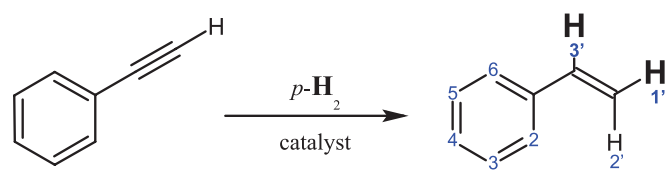

phenylacetylene styrene

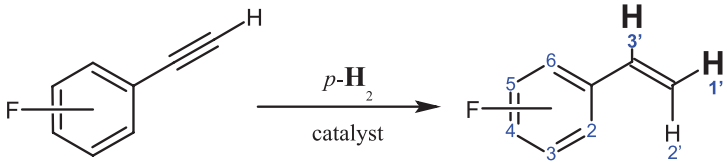

fluorophenylacetylene

fluorostyrene<smiles>CCOC(=O)C#Cc1ccccc1</smiles>

ethyl phenylpropiolate<smiles>CCO[PH](=O)/C=C/c1ccsc1</smiles>

ethyl cinnamate

Chart 1. Structure of fully protonated phenylacetylene and its 2-, 3-, or 4- mono-fluorophenyl derivatives used in the study; catalytic hydrogenation reactions are also shown in the Chart. At the bottom the corresponding reaction of ethyl phenylpropiolate is shown.

\subsection{Experimental setup}

For field-cycling NMR experiments we used a device described elsewhere $[18,19]$ that enables precise positioning of the whole NMR probe including the sample in the fringe field of the $7 \mathrm{~T} \mathrm{NMR}$ spectrometer magnet. This allows one to vary the field in the range between $0.5 \mathrm{~T}$ and $7 \mathrm{~T}$. To go to lower fields (down to Earth's field and even lower) we switched the current in additional coils placed under the cryomagnet. Field variation was done by mechanically moving the sample through the field by using a digitally controlled step-motor; thus, the time profile, $B(t)$, of field variation was known precisely.

The hydrogenation reaction was performed inside the NMR sample tube, which was modified for PHIP experiments at variable field. A scheme of the bubbling device is shown in Fig. 1. On the right hand side a schematic picture and a photo are presented; the photo was taken while bubbling the $\mathrm{H}_{2}$ gas through the NMR sample tube inside the NMR probehead. In order to provide efficient dissolution [22,23] of the $\mathrm{H}_{2}$ gas in the solvent we minimized the size of the bubbles. For this purpose a small cylinder made out of sintered glass was placed inside the NMR sample tube and fixed by melting it with a flame. A Teflon insert was glued to the bottom of the sample tube. This design allows one to place the NMR tube in a socket (bushing place) in the probehead in a very simple and fast way. Due to a strong capillary effect and a little gas overpressure (about 50 mbar) in the tubing line the fluid does not flow back from the ampoule, but remains there for a sufficient time between subsequent bubbling periods. A drawback of this design, however, is that it does not allow one rotating the NMR sample tube, which re- 


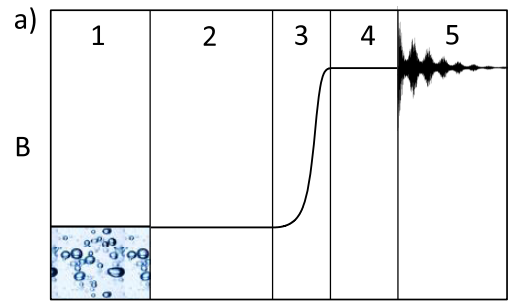

b)

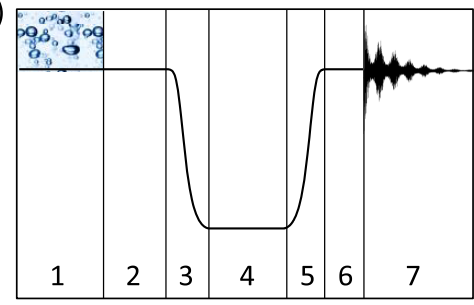

Time

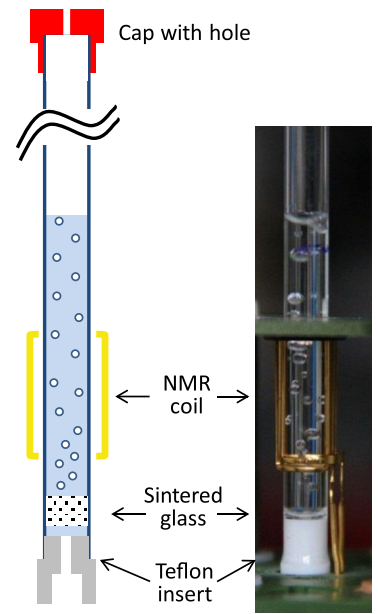

Fig. 1. Experimental protocols of PHIP experiments with one field jump (a) and two field jumps (b). On the right a scheme and a photo of the NMR sample tube for bubbling in situ are shown.

sults in a broadening of the NMR lines of about $2.5 \mathrm{~Hz}$. To control gas bubbling two magnetic valves (GSR Ventiltechnik $\mathrm{GmbH}$ ) were placed after the U-tube with charcoal that were operated by the computer control system of the NMR spectrometer. Opening the first valve starts to press gas through the solution. Using the second magnetic valve allows one to stop the parahydrogen injection quickly by releasing the overpressure. Otherwise, the gas continues to flow for approximately ten seconds, until the pressure drops to atmospheric pressure. The connection to the NMR tube is made out of flexible thin Teflon tubing, which allows one moving the sample tube position in the course of the field-cycling experiment. The delay between the control pulse and the actual start or stop of the $\mathrm{p}-\mathrm{H}_{2}$ injection was only $200 \mathrm{~ms}$.

This device enables bubbling of the $\mathrm{H}_{2}$ gas through the sample solvent in a controllable and reproducible way for many times. The method described is fully compatible with the magnetic field cycling: the probehead can be rapidly moved through the field and placed at any position in the fringe field of the NMR spectrometer with a precision of $0.1 \mathrm{~mm}$. The mechanical movement of the probehead does not have any observable effect on the reproducibility of the PHIP preparation.

\subsection{Experimental protocol}

To measure PHIP patterns as a function of the magnetic field we used the protocol shown in Fig. 1a, which consists of five steps. During the first two steps the polarized products of the hydrogenation are formed (PHIP preparation step of duration $\tau_{\mathrm{p}}$ ) at the magnetic field $B_{\mathrm{p}}$. In its turn, the preparation consists of two steps: during step 1 bubbling of the hydrogen gas through the solvent is performed for the time $\tau_{p 1}$; after this the hydrogenation reaction continues to run for the time $\tau_{p 2}$ producing polarized molecules (step 2); the sum of the two times is equal to $\tau_{\mathrm{p}}: \tau_{\mathrm{p}}=\left(\tau_{p 1}+\tau_{p 2}\right)$. The preparation times 
were taken rather long ( $\tau_{p 1}=15 \mathrm{~s}, \tau_{p 2}=20 \mathrm{~s}$ ) to guarantee that all coherences between the non-thermally populated states were washed out $[10,13]$. The reaction continues running; however, the experimental settings were chosen in such a way that the observed signals were coming almost exclusively from molecules polarized at field $B_{\mathrm{p}}$. Our setup provides high reproducibility of the preparation step: the line intensities in PHIP spectra taken under the same conditions scattered only by $5 \%$; however, the integral PHIP intensity shows a dependence on the number of repetitions: during the first 5-7 repetitions of bubbling PHIP grows, whereas during the following 8-30 repetitions PHIP slowly decreases. This behavior can be explained by activation of the catalysts followed by its degradation [24], but is not a subject of our interest. After having prepared the polarization the field is rapidly switched to the detection field $B_{0}=7 \mathrm{~T}$ of the NMR spectrometer during time $\tau_{\mathrm{fv}}$ (step 3, field variation). Time $\tau_{\mathrm{fv}}$ was always much shorter than $\tau_{\mathrm{p}}$, so PHIP produced at variable field during step 3 was negligible as compared to that produced at $B=B_{\mathrm{p}}$. The minimal time $\tau_{\mathrm{fv}}$ that we used for going from the lowest to the highest field was $0.41 \mathrm{~s}$. After going to $B=B_{0}$ and a short delay $\tau_{\text {dead }}$ (step 4, being a waiting time necessary to minimize mechanical vibration and field instability; usually $\tau_{\text {dead }}$ was $0.3 \mathrm{~s}$ ) the Fourier transform (FT) NMR spectrum is taken (step 5).

For manipulating PHIP in a more specific way we also used another protocol using two field jumps as depicted in Fig. 1b. This protocol comprises 7 consecutive stages. Polarized hydrogenation products are produced at a field $B=B_{\mathrm{p}}$ (steps 1-2); then during time $\tau_{\mathrm{fv} 1}$ the field is switched to the field of intermediate strength $B_{\text {int }}$ (step 3). At this field the spin system evolves freely during the waiting time $\tau_{\mathrm{w}}$ (step 4); then the field is switched back to $B_{0}$ during time $\tau_{\mathrm{fv} 2}$ (step 5) and after a short delay (step 6) the FT NMR spectrum is taken (step 7). As will be shown variation of the field $B_{\text {int }}$ and using three times $\tau_{\mathrm{fv} 1}, \tau_{\mathrm{w}}, \tau_{\mathrm{fv} 2}$ enables manipulating PHIP in a specific way to create a spin order of choice.

\section{Theory}

Theoretical modeling of the proton PHIP spectra was also performed. Here we will only briefly mention the main points of our theoretical approach, which is discussed in detail elsewhere $[10,13,25]$. In the simulations we calculated numerically the PHIP evolution in a system of $K$ coupled spins $1 / 2$. Because of the long preparation times we assume that at the end of the preparation period the spin system is described by the density matrix $\hat{\sigma}_{0}$, which has only diagonal matrix elements in the eigen-basis of the Hamiltonian $\hat{H}_{\mathrm{p}}$ that corresponds to $B=B_{\mathrm{p}}$ :

$$
\hat{H}_{\mathrm{p}}=2 \pi\left\{-\sum_{i=1}^{K} v_{i \mathrm{p}} \hat{\mathbf{I}}_{i z}+\sum_{i<j}^{K} J_{i j}\left(\hat{\mathbf{I}}_{i} \cdot \hat{\mathbf{I}}_{j}\right)\right\} .
$$

Here $v_{i \mathrm{p}}=\gamma_{i} B_{\mathrm{p}}\left(1+\delta_{i}\right) / 2 \pi$ is the Larmor precession frequency of the $i$-th spin that is given by its gyromagnetic ratio, $\gamma_{i}$, and its chemical shift, $\delta_{i} ; J_{i j}$ is the constant of scalar spin-spin interaction between the $i$-th and the $j$-th spins; $\hat{\mathbf{I}}_{i}$ is the spin operator of the $i$-th spin while $\hat{I}_{i z}$ is its $z$-component. In the eigen-basis of the Hamiltonian (1) the 
elements of $\hat{\sigma}_{0}$ are as follows:

$$
\sigma_{i j}=p_{i j} \delta_{i j}, \quad \text { where } \quad p_{i j}=\left\langle i\left|\hat{\sigma}_{s}^{(1,2)}\right| j\right\rangle \text { and } \delta_{i j} \text { is Kronecker's delta . }
$$

Here $|i\rangle$ and $|j\rangle$ denote the $i$-th and $j$-th eigen-states of $\hat{H}_{\mathrm{p}}$, respectively; $\hat{\sigma}_{s}^{(1,2)}$ is the density matrix of a state where spins 1 and 2 are in singlet correlation, while the other $(K-2)$ spins have no polarization. Spins originating from para-hydrogen will always be denoted as spin 1 and spin 2. For instance, for styrene molecules these are located at positions $\mathrm{H}^{\prime}$ and $\mathrm{H}^{\prime}$.

The evolution of the spin system during the subsequent field variations (in both protocols) is described by the following Liouville equation for the spin density matrix:

$$
\partial_{t} \hat{\sigma}=-i[\hat{H}(t), \hat{\sigma}]
$$

with the initial condition $\hat{\sigma}(t=0)=\hat{\sigma}_{0}$. The Hamiltonian of the system, which is timedependent during field variation, is as follows:

$$
\hat{H}(t)=2 \pi\left\{-\sum_{i=1}^{K} v_{i}(t) \hat{I}_{i z}+\sum_{i<j}^{K} J_{i j}\left(\hat{\mathbf{I}}_{i} \cdot \hat{\mathbf{I}}_{j}\right)\right\} .
$$

The precession frequencies $v_{i}(t)=\gamma_{i} B(t)\left(1+\delta_{i}\right) / 2 \pi$ change upon field variation. We solved Eq. (3) numerically by using the actual profile of field switching with taking into account the delay $\left(\tau_{\text {dead }}\right)$ at high field before applying the rf detection pulse and delays at the intermediate field (for both protocols shown in Fig. 1).

Once the density matrix after a field variation, $\hat{\sigma}_{\mathrm{fv}}$, was computed in this way we calculated the FT NMR spectrum in the same fashion as described in Refs. [10,13,2528] taking into account the rotation angle, $\varphi$, of the rf detection pulse. This step becomes important because of the non-trivial $\varphi$-dependence of the FT NMR spectra of non-thermally polarized systems: in contrast to thermally polarized systems the spectrum does not vary as $\sin (\varphi)$ but also has contributions that vary as $\sin (n \varphi)$ with $n \leq K[28,29]$. They are conditioned by higher spin orders, which are the expectation values of the products of spin operators. For instance, the single-spin order, which is the net polarization of the $i$-th spin, is the expectation value of $\hat{I}_{i z}:\left\{\hat{I}_{i z}\right\rangle=\operatorname{Tr}\left\{\hat{I}_{i z} \hat{\sigma}_{\mathrm{fv}}\right\}$ and gives contributions proportional to $\sin (\varphi)$. The two-spin order of the $i$-th and $j$-th spins, which is the multiplet polarization of the two spins, is the expectation value of the product operator $\hat{I}_{i z} \hat{I}_{j z}$ and gives contributions proportional to $\sin (2 \varphi)$ and so on. The $n$-spin order results in $\sin (n \varphi)$ contributions.

This simulation procedure allowed us to achieve good agreement between theory and experiment in the case of coupled protons. In the case of hetero-nuclei we could not simulate the spectra in a quantitative way because the spin systems were too complicated showing effects of cross-relaxation, and their NMR parameters, $\delta_{i}$ and $J_{i j}$, were not known in sufficient precision, which is a prerequisite for the quantitative simulation. Therefore, we restrict ourselves to a qualitative description of the PHIP transfer to ${ }^{13} \mathrm{C}$ and ${ }^{19} \mathrm{~F}$ nuclei. 


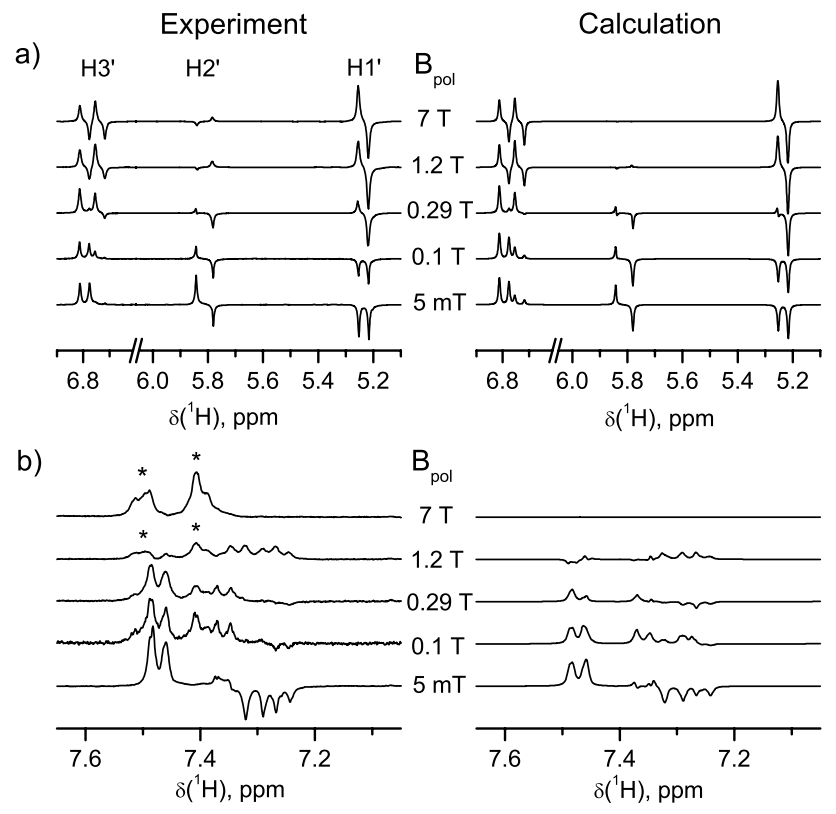

Fig. 2. Experimental and simulated PHIP spectra of styrene at different fields $B_{\mathrm{p}}$ : $\mathrm{H} 1^{\prime}, \mathrm{H} 2^{\prime}$ and $\mathrm{H}^{\prime}$ protons and aromatic protons. Subplot (a) shows results for the $\mathrm{H}^{\prime}, \mathrm{H} 2^{\prime}$ and $\mathrm{H} 3^{\prime}$ protons; subplot (b) shows results for the aromatic protons. Experiments were done according to protocol (a); $\tau_{\mathrm{fv}} \leq 0.41 \mathrm{~s}, \varphi=\pi / 4$, NMR parameters of styrene were taken from Table 1; the actual time profile of field variation was used in the calculation. Asterisks denote thermally polarized phenylacetylene.

\section{Results and discussion}

Now let us describe the experimental results. First, we will present the magnetic field dependence of PHIP in the coupled three-spin system of styrene and see how the ALTADENA-type spectrum at low fields is converted into the PASADENA-type spectrum as the field increases. Then we will demonstrate how PHIP in such a system can be manipulated in a controllable way by using two field jumps. Finally we will show that PHIP can be transferred to hetero-nuclei in a wide field range although the hetero-nuclei are strongly coupled with the protons only at very low fields.

\subsection{Field dependence of PHIP in a coupled three-spin system}

PHIP spectra of styrene taken for several polarization fields $B_{\mathrm{p}}$ are shown in Fig. 2; the field dependencies of PHIP are shown in Fig. 3. At high fields only the two protons originating from para-hydrogen, namely, the $\mathrm{H}^{\prime}$ and $\mathrm{H}^{\prime}{ }^{\prime}$ protons (spin 1 and spin 2 in our notations, see Eq. (3)), get polarized and the spectrum is of the PASADENAtype. The polarization of the two spins is of the multiplet type. The polarization of the third spin under PASADENA-conditions is much weaker and is presumably caused by cross-relaxation. As the field goes down the third spin also gets strongly polarized and the polarization pattern of the first two spins changes (Fig. 2a). The proton in the 
Table 1. NMR parameters of styrene used in calculations: chemical shifts and spin-spin interaction constants.

\begin{tabular}{lllllllll}
\hline $\begin{array}{l}\text { Protons and } \\
\text { chemical shift }\end{array}$ & $\mathrm{H} 1^{\prime}$ & $\mathrm{H} 2^{\prime}$ & $\mathrm{H} 3^{\prime}$ & $\mathrm{H} 2$ & $\mathrm{H} 3$ & $\mathrm{H} 4$ & $\mathrm{H} 5$ & $\mathrm{H} 6$ \\
\hline $\mathrm{H} 1^{\prime}, 5.236 \mathrm{ppm}$ & $\mathrm{X}$ & 1.054 & 10.941 & 0.158 & 0 & 0.281 & 0 & 0.158 \\
$\mathrm{H} 2^{\prime}, 5.812 \mathrm{ppm}$ & & $\mathrm{X}$ & 17.652 & 0 & 0 & 0.237 & 0 & 0 \\
$\mathrm{H} 3^{\prime}, 6.764 \mathrm{ppm}$ & & & $\mathrm{X}$ & -0.525 & 0.368 & -0.227 & 0.368 & -0.525 \\
$\mathrm{H} 2,7.470 \mathrm{ppm}$ & & & & $\mathrm{X}$ & 7.791 & 1.243 & 0.597 & 1.905 \\
$\mathrm{H} 3,7.345 \mathrm{ppm}$ & & & & & $\mathrm{X}$ & 7.445 & 1.422 & 0.597 \\
$\mathrm{H} 4,7.270 \mathrm{ppm}$ & & & & & & $\mathrm{X}$ & 7.445 & 1.243 \\
$\mathrm{H} 5,7.345 \mathrm{ppm}$ & & & & & & & $\mathrm{X}$ & 7.791 \\
$\mathrm{H} 6,7.470 \mathrm{ppm}$ & & & & & & & & $\mathrm{X}$ \\
\hline
\end{tabular}

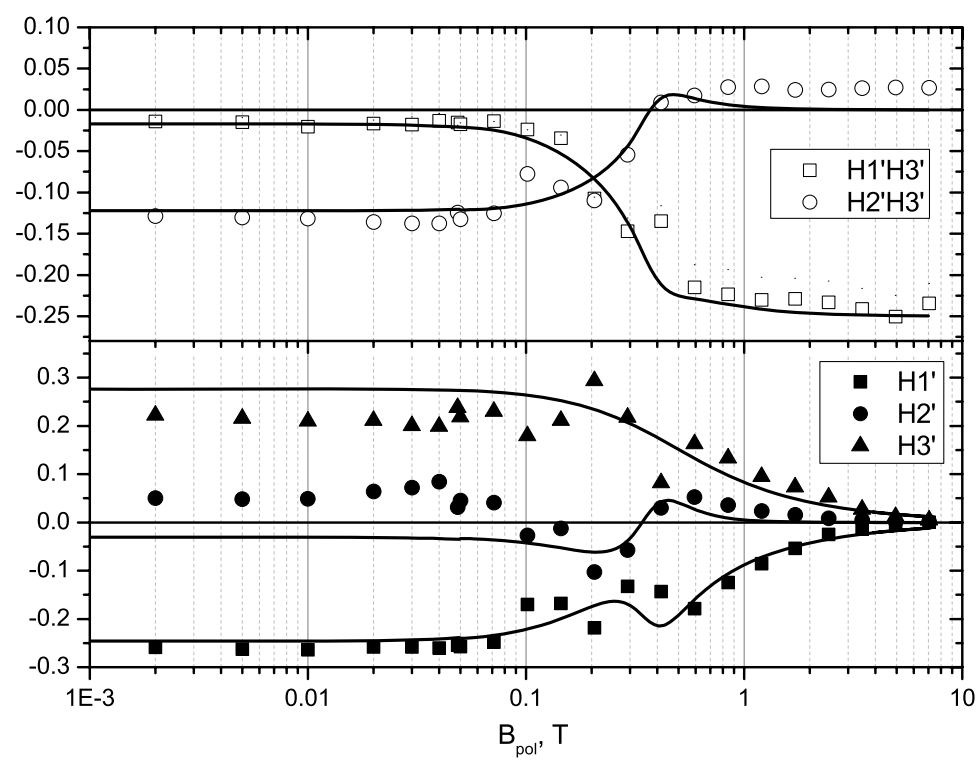

Fig. 3. Magnetic field dependence of PHIP for the $\mathrm{H} 1^{\prime}, \mathrm{H} 2^{\prime}$ and $\mathrm{H}^{\prime}$ protons of styrene. Lower subplot shows the field dependence of net polarization of the protons; upper subplot shows the multiplet polarization for two pairs of protons, $\mathrm{H}^{\prime}-\mathrm{H} 3^{\prime}$ and $\mathrm{H} 2^{\prime}-\mathrm{H}^{\prime}$. Solid lines show the calculation; the NMR parameters of styrene were taken from Table 1; the actual profile of field variation was used in the calculation. Experiments were done according to protocol (a); field variation time was $\tau_{\mathrm{fv}} \leq 0.41 \mathrm{~s}$.

$\mathrm{H} 3^{\prime}$-position acquires positive net polarization whereas the $\mathrm{H1}^{\prime}$ proton has negative net polarization. Such a polarization pattern is typical for ALTADENA experiments [25]: the spin with the largest chemical shift on the $\delta$-scale (H3' in our case) acquires positive polarization, while the spin with the smallest chemical shift ( $\mathrm{H}^{\prime}$ in our case) has negative polarization. In addition, PHIP transfer to other spins is present due to the strong coupling of the entire system. It is worth noting that the aromatic protons in styrene are 
also polarized at low magnetic fields where they are strongly coupled to the $\mathrm{H}^{\prime}, \mathrm{H} 2^{\prime}$ and $\mathrm{H}^{\prime}$ protons.

Most of the experimental spectra (see Fig. 2) are in very good agreement with the theoretical simulation, which is a clear indication that our approach can model PHIP at variable magnetic field. The NMR parameters of styrene [30] that were used for simulation are given in Table 1 . Theory can describe the gradual change from the ALTADENA-like to the PASADENA-like spectral pattern upon raising the field and the polarization transfer to the third proton (Fig. 2a). Although in the simulations it is preferable to use the actual time profile $B(t)$ of field variation in the case under study the low field PHIP spectrum is also reproduced under the assumption of slow (adiabatic) switching (not shown here). This is an indication that in this particular case the state populations of the adiabatic energy levels stay almost constant; therefore a close correlation of the low-field and the high-field states is given. However, in other cases it becomes necessary [13] to take the actual switching profile into account.

We studied PHIP not only of the $\mathrm{H}^{\prime}, \mathrm{H}^{\prime}$ and $\mathrm{H}^{\prime}$ protons of styrene but also of the rather weakly polarized aromatic protons. PHIP transfer to the aromatic protons is possible due to strong coupling of the entire spin system at low magnetic fields. The aromatic parts of the PHIP spectra are shown in Fig. 2b. We performed theoretical modeling of the PHIP spectra assuming that the transfer mechanism is provided by scalar spin-spin interactions. In the calculations we considered the entire coupled eight-spin system. The results of the theoretical modeling are in good agreement with the experimental data at all magnetic fields. Polarization transferred to the aromatic protons is predominantly net polarization except for low fields $\left(e . g ., B_{\mathrm{p}}=5 \mathrm{mT}\right)$ where only multiplet polarization can be formed. The good agreement between theory and experiment is an indication that the main mechanism of PHIP re-distribution in the molecule is coherent polarization transfer caused by strong coupling among spins at low magnetic fields.

Not only the spectral patterns, but also the field dependence of PHIP is reproduced by our calculation. In Fig. 3 the field dependence of the net polarizations, $\left\langle I_{\mathrm{H}^{\prime} z}\right\rangle,\left\langle I_{\mathrm{H}^{\prime} z}\right\rangle$ and $\left\langle I_{\mathrm{H} 3^{\prime} z}\right\rangle$, and of the multiplet polarizations, $\left\langle I_{\mathrm{H}^{\prime} z} I_{\mathrm{H}^{\prime} z}\right\rangle$ and $\left\langle I_{\mathrm{H}^{\prime} z} I_{\mathrm{H} 3^{\prime} z}\right\rangle$, is shown.

Each $\left\langle I_{i z}\right\rangle$ was obtained by integration over the corresponding spin multiplet in the NMR spectrum. As the field increases $\left\langle I_{\mathrm{H}^{\prime} z}\right\rangle$ and $\left\langle I_{\mathrm{H}^{\prime} z}\right\rangle$ gradually go to zero from the initial negative and positive values, respectively. This corresponds to transition from ALTADENA (individual spins exhibit net polarization) to the PASADENA case (only multiplet polarization is left). The net polarization $\left\langle I_{\mathrm{H} 2^{\prime} z}\right\rangle$ is always close to zero.

The value of $\left\langle I_{i z} I_{j z}\right\rangle$ was obtained by taking the difference in intensity of the lines within the same NMR multiplet. We were not able to measure the multiplet effect $\left\langle I_{\mathrm{H} 1^{\prime} z} I_{\mathrm{H} 2^{\prime} z}\right\rangle$ because of the tiny spin-spin interaction (about $1 \mathrm{~Hz}$ ) between the $\mathrm{H}^{\prime}$ and $\mathrm{H} 2^{\prime}$ protons, which did not allow us to separate the corresponding lines within the multiplets. The values of $\left\langle I_{\mathrm{H}^{\prime} z} I_{\mathrm{H} 3^{\prime} z}\right\rangle$ and $\left\langle I_{\mathrm{H} 2^{\prime} z} I_{\mathrm{H} 3^{\prime} z}\right\rangle$ were reliably determined for all fields $B_{\mathrm{p}}$; their field dependence is also shown in Fig. 3 (upper part). At low field the multiplet polarization $\left\langle I_{\mathrm{H}^{\prime} z} I_{\mathrm{H}^{\prime} z}\right\rangle$ is considerably larger than $\left\langle I_{\mathrm{H}^{\prime} z} I_{\mathrm{H}^{\prime} z}\right\rangle$; however, it goes to zero as the field increases. At high fields (weakly coupled spins) only the multiplet polarization $\left\langle I_{\mathrm{H}^{\prime} z} I_{\mathrm{H}^{\prime} z}\right\rangle$ remains, which becomes equal to $-1 / 4$. This corresponds to the PASADENA situation, while at low fields (strongly coupled spins) all spin orders are pronounced due to the polarization transfer in the molecule. 
At magnetic fields around $B_{\text {lac }} \approx 0.35 \mathrm{~T}$ there is a sharp feature seen in the dependences of $\left\langle I_{\mathrm{H}^{\prime} z}\right\rangle$ and $\left\langle I_{\mathrm{H}^{\prime} z}\right\rangle$ and, in particular, in the dependences of the multiplet effect (sharp increase of $\left\langle I_{\mathrm{H}^{\prime} z} I_{\mathrm{H}^{\prime} z}\right\rangle$ and sharp decrease of $\left.\left\langle I_{\mathrm{H}^{\prime} z} I_{\mathrm{H}^{\prime} z}\right\rangle\right)$. They are caused by a level anti-crossing (LAC) in the strongly coupled three-spin system, which is always expected to cause features in the field dependence of polarization in coupled spin systems $[10-12,17]$. Although in the present case this feature is not very pronounced it can lead to efficient polarization transfer in the spin system once the parameters of field variation or the experimental protocol are changed. In particular, it is possible to polarize the third spin by going through the level anti-crossing with an appropriately set speed. This is demonstrated in the following subsection.

\subsection{PHIP in experiments with two field jumps}

To demonstrate the LAC effect more clearly we ran experiments using the second protocol shown in Fig. 1b that takes advantage of two field jumps. PHIP was prepared at high magnetic field $B_{0}=7 \mathrm{~T}$ where the spins are coupled weakly with the consequence that only the first two protons directly polarized by PHIP acquired considerable polarization (PASADENA spectral pattern). Then the field was rapidly switched down to the intermediate level, $B_{\text {int }}$ and after a short delay $\tau_{\mathrm{w}}$ (about $50 \mathrm{~ms}$ ) switched again to $B_{0}$ in order to detect the polarization of the spin system. In this case there can be coherent and efficient exchange of population between the spin energy levels. The prerequisite for such a population exchange is as follows: one has to vary the field from the weak to the strong coupling regime using fast switching (sudden). When these conditions are fulfilled the population difference between the two coupled levels at high field is transformed into coherence between them at low field. The time evolution of the coherence leads to exchange between different spin orders, which is much faster and more efficient than cross-relaxation. In the case under consideration such coherent processes can mix the initial singlet spin order with net polarization of the spins. The second switching to the field $B_{0}$ is necessary not only for recording the NMR spectrum but also for transforming the coherence present in the system at low field back into a population difference. Setting properly the timing of such experiment allows one to manipulate efficiently the transfer process. To verify this idea we performed numerical calculations of spin evolution under the influence of two field variations.

Experimental and theoretical results are shown in Fig. 4 for several magnetic fields. The polarization pattern does not change from the PASADENA pattern when $B_{\text {int }}>B_{\text {lac }}$ because for the chosen parameters the condition of sudden switching can only be achieved for a pair of anti-crossing levels when the system passes through the crossing point. Once the $B_{\text {int }}$ field is above the level crossing region there is no mixing between the levels. When $B_{\text {int }}<B_{\text {lac }}$ there is mixing only between the two crossing levels because the condition of sudden variation of the Hamiltonian is fulfilled only for this pair of levels. It results in a highly efficient conversion of the initial singlet order, $\left\langle I_{\mathrm{H}^{\prime} z} I_{\mathrm{H}^{\prime} z}\right\rangle$, into net polarization of the individual spins. In particular, in the example chosen the multiplet polarization $\left\langle I_{\mathrm{H}^{\prime} z} I_{\mathrm{H}^{\prime} z}\right\rangle$ is to a very high extent converted into the net polarizations $\left\langle I_{\mathrm{H}^{\prime} z}\right\rangle$ and $\left\langle I_{\mathrm{H}^{\prime} z}\right\rangle$ due to the dynamic mixing of the anti-crossing levels. As a consequence, the third spin, which is not polarized at high field, acquires considerable net PHIP. More specifically, the multiplet $\left\langle I_{\mathrm{H}^{\prime} z} I_{\mathrm{H}^{\prime} z}\right\rangle$ polarization is trans- 
Experiment

Calculation

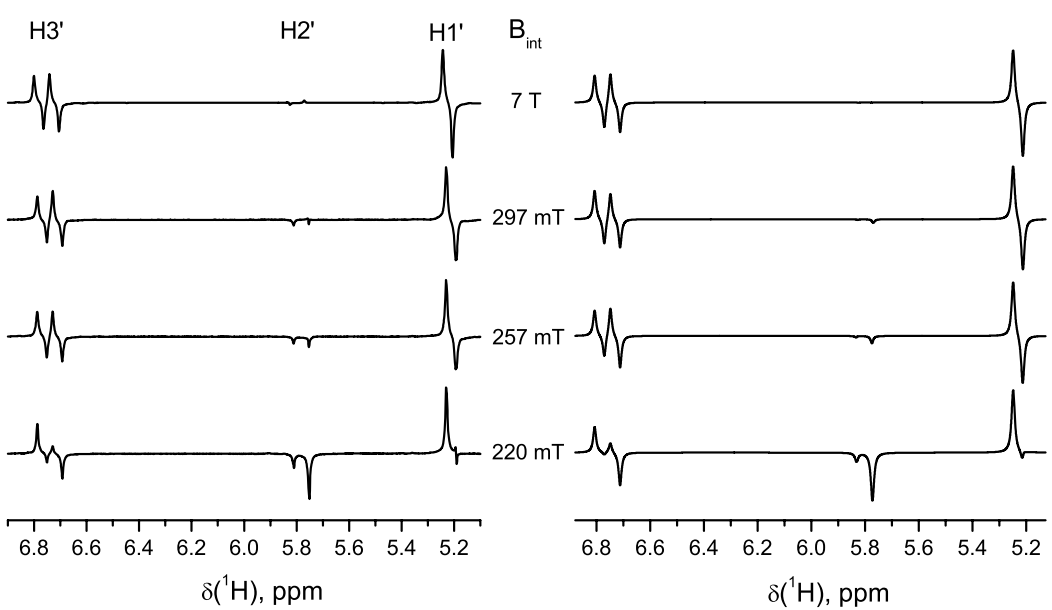

Fig. 4. Experimental and simulated PHIP spectra of the $\mathrm{H} 1^{\prime}, \mathrm{H} 2^{\prime}$ and $\mathrm{H}^{\prime}$ protons of styrene at different fields $B_{\text {int }}$. Experiments were done according to protocol (b): $\tau_{\mathrm{fv}} \leq 0.41 \mathrm{~s}, \varphi=\pi / 4, B_{\mathrm{p}}=B_{0}=7 \mathrm{~T}$; NMR parameters of styrene were taken from Table 1; the actual time profile of field variation was used in the calculation.

formed into the positive $\left\langle I_{\mathrm{H}^{\prime} z}\right\rangle$ and the negative $\left\langle I_{\mathrm{H}^{\prime} z}\right\rangle$ net polarization. This is because the two eigen-levels, which exchange populations at low $B_{\text {int }}$, correspond to the $|\alpha \alpha \beta\rangle$ and $|\beta \alpha \alpha\rangle$ high-field states. One of these states, namely, $|\beta \alpha \alpha\rangle$ (involving the opposite $z$-projections of spins originating from para-hydrogen), is overpopulated due to PHIP prior to the field switching, whereas $|\alpha \alpha \beta\rangle$ has zero population. After coherent dynamic mixing the state $|\alpha \alpha \beta\rangle$ becomes over-populated. The sign of the $z$-projections of the spins ( $\alpha$ for the first spin and $\beta$ for the third spin) in this state results in the positive net polarization $\left\langle I_{\mathrm{H}^{\prime} z}\right\rangle$ and the negative net polarization $\left\langle I_{\mathrm{H}^{\prime} z}\right\rangle$. For the field variation parameters chosen at $B_{\text {int }}=220 \mathrm{mT}$ the conversion of $\left\langle I_{\mathrm{H}^{\prime} z} I_{\mathrm{H}^{\prime} z}\right\rangle$ to negative $\left\langle I_{\mathrm{H}^{\prime} z}\right\rangle$ is highly pronounced. As it is seen from the corresponding spectrum the multiplet polarization $\left\langle I_{\mathrm{H}^{\prime} z} I_{\mathrm{H}^{\prime} z}\right\rangle$, which is the difference in intensities of the two lines of the $\mathrm{H} 3^{\prime}-$ proton, is reduced by a factor of approximately two with respect to the PASADENA case, while the spin order is efficiently converted into negative net polarization of the $\mathrm{H} 2$ '-proton. At all fields the $\mathrm{H}^{\prime}$-proton has only multiplet polarization. After two field jumps two lines of the multiplet are reduced but the character of polarization stays the same. Thus, exploiting the LAC one can efficiently manipulate PHIP by selectively funnelling it to particular spin orders of choice. It is important to note that the optimal field for conversion of the spin order is not necessarily $B_{\text {lac }}$. The optimal field is always below $B_{\text {lac }}$ because a double rapid passage through the LAC is required for efficient exchange of populations of the crossing levels. During the passage from $B_{\text {lac }}$ to $B_{\text {int }}$ and back there is coherent mixing occurring between the crossing levels. Precise prediction of the optimal parameters is possible only by using numerical calculations because the $B(t)$ profile is relatively complicated as the field variation speed, $d B(t) / d t$, is not constant during a single passage. As in the previous case our theoretical model can describe the 
observed spectra very well (Fig. 4). Effects of converting the initial singlet spin order $\left\langle I_{\mathrm{H}^{\prime} z} I_{\mathrm{H} 3^{\prime} z}\right\rangle$ into the negative net polarization $\left\langle I_{\mathrm{H}^{\prime} z}\right\rangle$ are perfectly reproduced.

\subsection{Transfer of PHIP to ${ }^{13} \mathrm{C}$ and ${ }^{19} \mathrm{~F}$}

In this section let us demonstrate how PHIP can be transferred from protons to spin $1 / 2$ hetero-nuclei.

In general, polarization transfer among spins requires their strong coupling. The strong coupling condition can be easily fulfilled for two protons that often are strongly coupled even at high fields of a few Tesla. But for strong coupling between protons and hetero-nuclei very low fields are required, which are of the order of $10 \mu \mathrm{T}$ and usually even lower. Going to such low fields can be rather demanding technically although feasible $[31,32]$. However, such low fields are only needed to create net polarization of the hetero-nucleus whereas other spin orders can be formed at much higher fields, which are much easier to achieve [25,33]. Polarization transfer to hetero-nuclei proceeds in a similar way also in reversible reactions of substrates with para-hydrogen [16]. In particular, it is possible to transfer multiplet polarization to the hetero-nuclei from the protons originating from para-hydrogen. The conditions for creating such spin order are much less demanding: (i) the two protons must be strongly coupled with each other at the preparation field $B_{\mathrm{p}}$ and (ii) they must have non-equal couplings to the heteronucleus. In practice, both conditions can be easily achieved: this type of polarization of the hetero-nucleus can be formed at magnetic fields even up in the Tesla range. The origin of such polarization is as follows: the size of the net polarization, $\left\langle I_{1 z}\right\rangle$ and $\left\langle I_{2 z}\right\rangle$, of strongly coupled protons depends on the difference, $\delta v$, in their Zeeman interaction with the field [25,34]. The hetero-nucleus shifts this difference by $\delta J \cdot F_{z}$ where $\delta J$ is the difference of the two hetero-nuclear spin-spin interactions and $F_{z}$ is the z-projection of the hetero-spin. Once $\delta J \neq 0$ the $\delta v$ value is different for different states of the heteronucleus. As a consequence, the $\left\langle I_{1 z}\right\rangle$ and $\left\langle I_{2 z}\right\rangle$ values depend on $F_{z}$, which means that proton-heteronuclear polarization is formed.

The idea of creating multiplet polarization of the protons and the hetero-nucleus was proposed in Ref. [33] while analytical results for PHIP in the simple three-spin system (two protons, which originate from para-hydrogen, are weakly and unequally coupled to the spin 1/2 hetero-nucleus) have been obtained in Ref. [25]. However, experimentally this idea was never investigated thoroughly. In this work we perform a study of PHIP transfer to ${ }^{13} \mathrm{C}$ and ${ }^{19} \mathrm{~F}$ nuclei based on the mechanism described above at different polarization fields $B_{\mathrm{p}}$. We have studied PHIP effects in fluorinated styrene molecules having one fluorine atom in the aromatic ring at different positions and also in ethyl-cinnamate with natural abundance of ${ }^{13} \mathrm{C}$ atoms. Here we present only experimental results for all the systems studied. We also performed theoretical modeling of PHIP effects in 4-fluorostyrene; however, agreement between the experimental and calculation results was considerably worse than in the case of styrene. A possible reason for this is rather efficient cross-relaxation, which can compete with the coherent PHIP re-distribution. We were not able to verify this idea theoretically because of the complexity of the system, which comprises eight coupled spins. An extension of the theoretical treatment of the problem that takes the relaxation effects into account is beyond the scope of the present work and will be performed later. We did not per- 


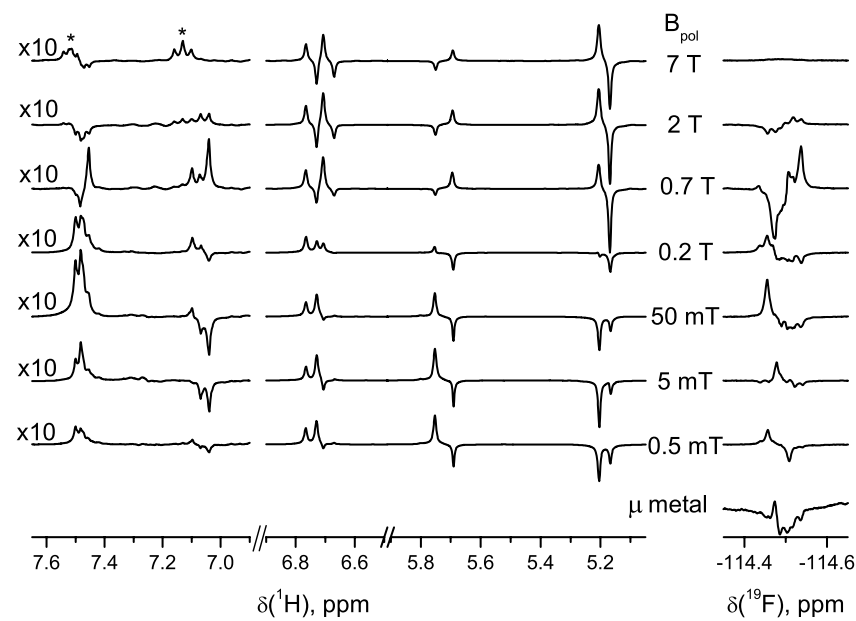

Fig. 5. Experimental ${ }^{1} \mathrm{H}$ and ${ }^{19} \mathrm{~F}$ PHIP spectra of 4-fluorostyrene at different magnetic fields $B_{\mathrm{p}}$. Experiments were done according to protocol (a); $\tau_{\mathrm{fv}} \leq 0.6 \mathrm{~s}, \varphi=\pi / 4$. Asterisks denote signals from thermally polarized 4-fluorophenylacetylene. A ${ }^{19} \mathrm{~F}$ PHIP spectrum obtained after placing the sample in a $\mu$-metal box is also shown.

form the theoretical modeling for the other molecules (2-fluorostyrene, 3-fluorostyrene and ethyl-cinnamate) because of the complexity of the network of couplings and the incomplete knowledge of their NMR parameters.

PHIP transfer to ${ }^{19} \mathbf{F}$ nuclei. Spectra of 4-fluorostyrene are shown in Fig. 5 at different fields $B_{\mathrm{p}}$; both proton and fluorine spectra are presented. The spectrum of the $\mathrm{H}^{\prime}, \mathrm{H} 2^{\prime}$ and $\mathrm{H}^{\prime}$ ' protons shows characteristic changes from the ALATDENA-type to the PASADENA-type pattern upon increase of the field. At fields lower than $1 \mathrm{~T}$ the aromatic protons also get polarized because of the strong coupling of the entire proton system. However, the polarization of the aromatic protons is considerably lower than that of the $\mathrm{H} 1^{\prime}, \mathrm{H} 2^{\prime}$ and $\mathrm{H} 3^{\prime}$ protons. Our previous experimental studies and theoretical modeling [13] confirm that in similar systems strong coupling of spins is indeed the mechanism of PHIP transfer to the aromatic protons.

At magnetic fields up to $2 \mathrm{~T}$ we also observed strong non-thermal polarization of ${ }^{19} \mathrm{~F}$ nuclei. This polarization is of the multiplet type: the integral over all lines in the spectrum is zero but the individual lines are strongly enhanced. We explain polarization of such type as multiplet proton-fluorine polarization coming from the strongly coupled polarized protons at $B=B_{\mathrm{p}}$ and their unequal couplings to the fluorine spin. In our case it is problematic to discriminate between the following two possibilities: (i) PHIP is directly transferred to ${ }^{19} \mathrm{~F}$ from the protons originating from para- $\mathrm{H}_{2}$; (ii) PHIP is first transferred to the aromatic protons by means of their strong coupling to the $\mathrm{H} 1^{\prime}, \mathrm{H} 2^{\prime}$ and $\mathrm{H}^{\prime}$ protons and subsequently to ${ }^{19} \mathrm{~F}$. At the moment we are not able to verify, which of the mechanisms is operative in our case or whether (iii) both mechanisms are present, because of the complexity of the spin system under study. A rough estimate based on the NMR parameters of the spin system shows that, in principle, both of them can be operative. 


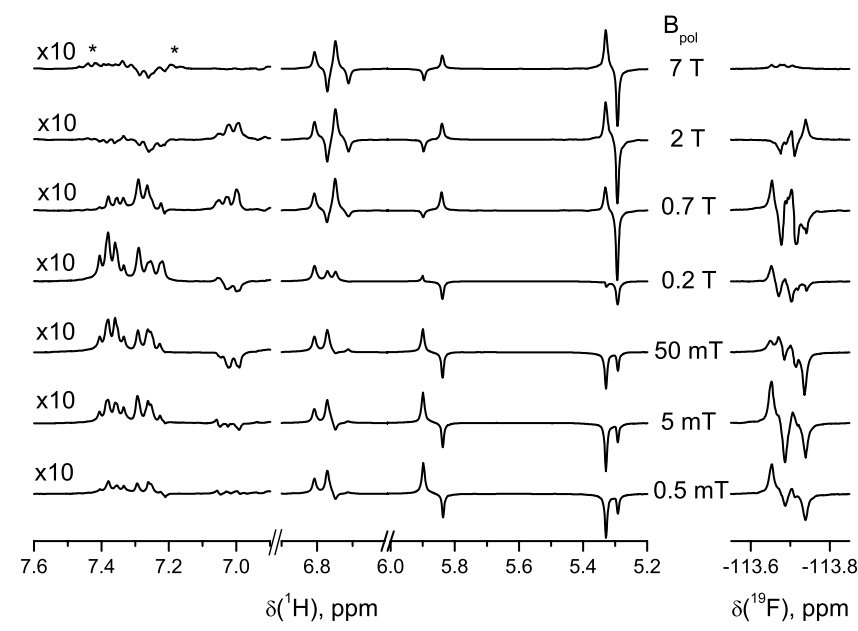

Fig. 6. Experimental ${ }^{1} \mathrm{H}$ and ${ }^{19} \mathrm{~F}$ PHIP spectra of 3-fluorostyrene at different magnetic fields $B_{\mathrm{p}}$. Experiments were done according to protocol (a); $\tau_{\mathrm{fv}} \leq 0.6 \mathrm{~s}, \varphi=\pi / 4$. Asterisks denote signals from thermally polarized 3-fluorophenylacetylene.

It is worth noting that in all cases the observed ${ }^{19} \mathrm{~F}$ polarization was of the multiplet type. It was possible to observe net PHIP of the ${ }^{19} \mathrm{~F}$ nucleus only when during the preparation period the sample was placed in a $\mu$-metal box as has been proposed earlier [31,32]. In this situation the spin system resides in ultra-low magnetic field for some time with the consequence that the proton and fluorine spins are strongly coupled and efficiently exchange their net polarization. As a consequence, net PHIP of ${ }^{19} \mathrm{~F}$ was observed (the corresponding fluorine PHIP spectrum is shown at the bottom of Fig. 5). However, once the magnetic field was comparable with the Earth's field or higher only multiplet proton-fluorine PHIP was observed.

Corresponding spectra of 3-fluorostyrene are shown in Fig. 6. The polarization of the proton sub-system looks very similar to the 4-fluorostyrene case. It is seen that the ${ }^{19} \mathrm{~F}$ spectra have a slightly different spectral pattern but in general we see only multiplet polarization transfer at fields less than $2 \mathrm{~T}$ and no polarization transfer to ${ }^{19} \mathrm{~F}$ at $7 \mathrm{~T}$. This is not the case for the 2-fluorostyrene (Fig. 7). On top of the multiplet polarization transfer there is net polarization on ${ }^{19} \mathrm{~F}$ nuclei in a wide field range from $0.05 \mathrm{~T}$ to 7 T. Such effects can be explained by inter molecular dipole-dipole cross-relaxation between protons and fluorine nuclei. These effects are expected to be most efficient for 2-fluorostyrene, since the ${ }^{19} \mathrm{~F}$ nucleus has the shortest distance to the directly polarized $\mathrm{H}^{\prime}$ ' and $\mathrm{H}^{\prime}$ ' protons. Cross-relaxation effects should also manifest themselves for the aromatic protons; however, this effect is expected to be less efficient than polarization transfer due to the scalar couplings.

Thus, the results presented in Figs. 5-7 demonstrate the possibility of creating strong non-thermal net polarization of the hetero-nuclei over a relatively broad magnetic field range. It should be mentioned that our results contradict observations of Kuhn et al. [20] who observed predominantly emissive net polarization of fluorine in 2-, 3- and 4-fluorostyrene at low $B_{\mathrm{p}}$. The reason of such a discrepancy is not clear and 


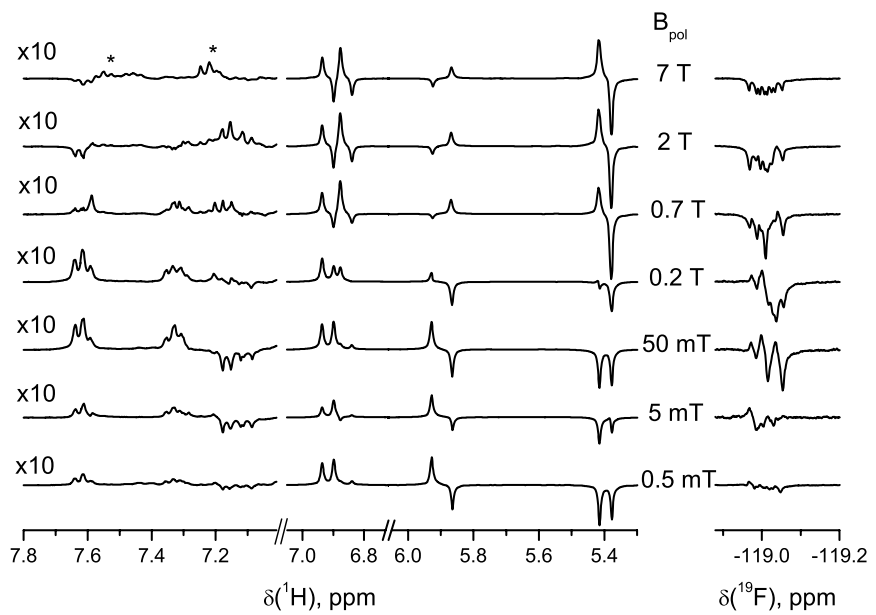

Fig. 7. Experimental ${ }^{1} \mathrm{H}$ and ${ }^{19} \mathrm{~F}$ PHIP spectra of 2-fluorostyrene at different magnetic fields $B_{\mathrm{p}}$. Experiments were done according to protocol (a); $\tau_{\mathrm{fv}} \leq 0.6 \mathrm{~s}, \varphi=\pi / 4$. Asterisks denote signals from thermally polarized 2-fluorophenylacetylene.

may be caused by cross-relaxation in the polarized molecules during the field variation. In our case field variation was performed so fast that it could not result in a considerable PHIP transfer to ${ }^{19} \mathrm{~F}$. In addition, our preparation field, $B_{\mathrm{p}}$, and field variation profile, $B(t)$, were precisely controlled. Since we did not observe similar polarization patterns cross-relaxation is a plausible mechanism of the hetero-nuclear PHIP transfer effects reported earlier [20].

Our study clearly demonstrates that it is possible to efficiently polarize heteronuclei by means of PHIP at relatively high strength of the external magnetic field. Although the polarization of the hetero-nuclei integrated over the whole spectrum is then zero the individual lines are very strongly enhanced. In principle, by applying appropriate pulse sequences this spin order can be converted to net polarization of hetero-nuclei [35-39]. This can be useful for NMR and, particularly, MRI applications for improving the image contrast and sensitivity. In this context it is important to discuss PHIP transfer to insensitive spin 1/2 hetero-nuclei, which have long relaxation times up to a few minutes. For this reason we studied PHIP transfer to ${ }^{13} \mathrm{C}$ nuclei.

PHIP transfer to ${ }^{13} \mathbf{C}$ nuclei. We studied PHIP effects in ethyl-cinnamate with $1.1 \%$ natural abundance of ${ }^{13} \mathrm{C}$. Carbon PHIP spectra are shown in Fig. 8. From a comparison with the NMR spectrum of the thermally polarized molecules it is readily seen that PHIP gives very large enhancements of ${ }^{13} \mathrm{C}$ signals. Carbon spins in almost all positions in the molecule except the ethyl group acquire strong PHIP, which is of pure multiplet character: each absorptive line is compensated by an emissive spectral component. We propose the following explanation for these observations. Because of the low natural abundance of ${ }^{13} \mathrm{C}$ nuclei each carbon position in the NMR spectrum corresponds to a different molecule with only one ${ }^{13} \mathrm{C}$ atom in the corresponding position. The probability that molecules have two and more 13 -carbons is smaller than $0.6 \%$. For this reason one can neglect intra-molecular carbon-carbon spin interactions and the situ- 


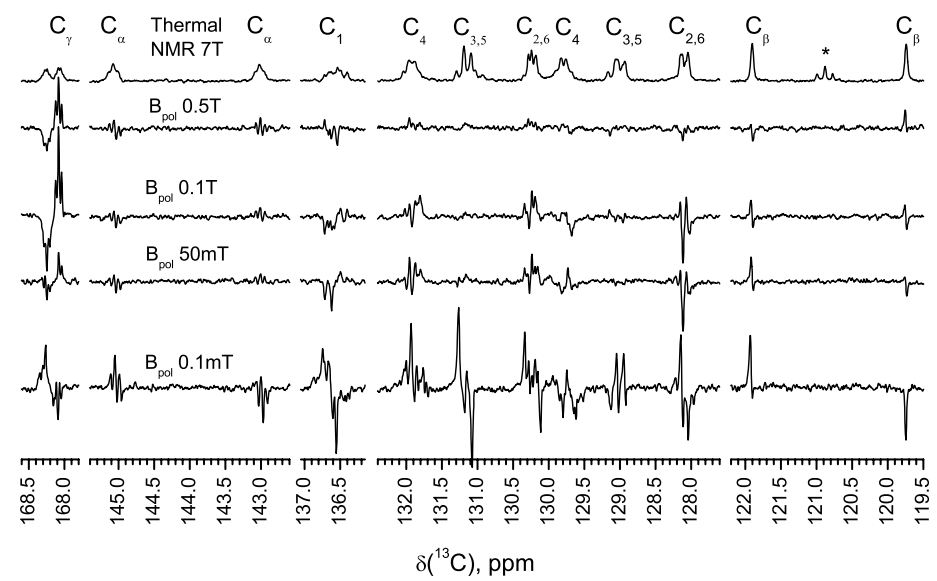

Fig. 8. Experimental ${ }^{13} \mathrm{C}$ PHIP spectra of ethyl-cinnamate $\left({ }^{13} \mathrm{C}\right.$ in natural abundance, 14 accumulations) at different magnetic fields $B_{\mathrm{p}}$. Experiments were done according to protocol (a); $\tau_{\mathrm{fv}} \leq 0.41 \mathrm{~s}, \varphi=\pi / 4$. ${ }^{13} \mathrm{C}$ NMR spectra of thermally polarized ethyl-cinnamate at natural carbon abundance obtained at $7 \mathrm{~T}$ after 12000 accumulations is shown for comparison. Asterisks denote signals from thermally polarized ethyl phenylpropiolate.

ation becomes similar to the case of polarized molecules with a single ${ }^{19} \mathrm{~F}$ nucleus. As consequence, carbons are polarized due to the same mechanism: each ${ }^{13} \mathrm{C}$ is unequally coupled to strongly coupled protons, which are polarized initially. Therefore the resulting polarization has carbon-proton multiplet character and can be observed in a broad field range for all carbon atoms in the molecule. We believe that this explanation is also valid for previous studies [20,40] where PHIP transfer to multiplet polarization of protons and hetero-nuclei was reported.

In summary, we observed PHIP transfer from protons to ${ }^{13} \mathrm{C}$ and ${ }^{19} \mathrm{~F}$ hetero-nuclei over a wide range of external magnetic fields. This transfer proceeds due to spin-spin interactions and is possible when the protons are strongly coupled with each other and have different couplings to the hetero-nucleus. The resulting hetero-nuclear polarization, which is of the multiplet type, can be formed in a way that is not particularly demanding from the technical viewpoint. The mechanism does not require going to very low magnetic fields. The size of the proton-heteronuclear multiplet polarization is large; we did not calibrate its size, but estimate that it is comparable to the initial proton polarization provided by PHIP. We expect that the mechanism described above is general and applies to other spin $1 / 2$ hetero-nuclei as well, for instance, to ${ }^{15} \mathrm{~N}$ and ${ }^{31} \mathrm{P}$. This type of polarization can be applied for obtaining anomalous enhancement of the NMR signals of insensitive nuclei.

\section{Conclusions}

In the present work we have studied transfer of PHIP based on strong coupling of spins at low fields. To gain a better insight in the phenomena under investigation we per- 
formed experiments at variable strength of the external magnetic field. We have studied experimentally and modelled theoretically PHIP effects in a three-spin system. At low fields we observed substantial transfer of polarization from protons originating from para-hydrogen to the third spin. In the field dependence of polarization we observed features caused by the LAC in the three-spin system. We were also able to model the PHIP re-distribution in the entire styrene molecule considering a coupled eight-spin $1 / 2$ system. To demonstrate the effects of the LAC with better clarity we modified the experimental protocol introducing two fast field jumps and observed efficient and selective transfer of the initial singlet order of two spins to net polarization of the third spin.

We also demonstrated experimentally effects of PHIP transfer to spin $1 / 2$ heteronuclei, namely to ${ }^{13} \mathrm{C}$ and ${ }^{19} \mathrm{~F}$. We have shown that in contradiction to general belief efficient PHIP transfer to hetero-nuclei does not require going to ultra-low fields of $10 \mu \mathrm{T}$ or less. We have observed polarization transfer to both ${ }^{13} \mathrm{C}$ and ${ }^{19} \mathrm{~F}$ in a wide field range. Although the resulting polarization has no net but multiplet character the method is advantageous because it allows one to obtain strong signal enhancements for NMR signals of ${ }^{13} \mathrm{C}$ and ${ }^{19} \mathrm{~F}$ without special equipment needed for ultra-low field experiments.

Our results show that field variation is a powerful tool in manipulating PHIP when one reaches the regime of strong coupling among spins. In particular, we expect that by exploiting level anti-crossings and controlling the time profile of field variation one can achieve particularly efficient exchange between specific spin orders. Adjusting the field properly also allows for optimizing the transfer of PHIP to particular spin orders of choice. Field variation also makes polarization transfer to hetero-nuclei possible. The mechanism of transfer has been explained; it was shown that it is operative even at relatively high magnetic fields (up to a few Tesla in the examples shown).

Although all the results were obtained for a particular type of non-thermal polarization, namely, for PHIP, our conclusions are much more general. They are not restricted to the technique that we used but apply to other types of hyperpolarization as well. In general, strong coupling among spins, which can be reached by appropriately lowering the field, is always a cause of efficient polarization transfer. Methods of manipulating spin polarization by going through a level anti-crossing are applicable to any type of polarization. Therefore, transfer of non-thermal polarization to low-gamma nuclei can be realized by using other polarization techniques as well.

\section{Acknowledgement}

Financial support by the Russian Fund for Basic Research (RFBR project No. 1103-00356, 11-03-00296), Program of the Division of Chemistry and Material Science RAS (project No. 5.1.1) and the Program P-220 of the Russian Government (grant No. 11.G34.31.0045) is gratefully acknowledged. We also thank the Alexander von Humboldt Foundation for a fellowship at FU-Berlin (A. S. K.), and for financial support via the research group linkage program (K. L. I., H.-M. V. and A. V. Y.). We are thankful to A. N. Pravdivtsev (NSU) for his help in modelling the PHIP field dependence. 


\section{References}

1. S. B. Duckett and C. J. Sleigh, Prog. NMR Spectrosc. 34 (1999) 71.

2. J. Natterer and J. Bargon, Prog. NMR Spectrosc. 31 (1997) 293.

3. K. Münnemann and H.-W. Spiess, Nature Phys. 7 (2011) 522.

4. R. W. Adams, J. A. Aguilar, K. D. Atkinson, M. J. Cowley, P. I. P. Elliott, S. B. Duckett, G. G. R. Green, I. G. Khazal, J. Lopez-Serrano, and D. C. Williamson, Science 323 (2009) 1708.

5. M. G. Pravica and D. P. Weitekamp, Chem. Phys. Lett. 145 (1988) 255.

6. C. R. Bowers and D. P. Weitekamp, J. Am. Chem. Soc. 109 (1987) 5541.

7. G. L. Closs and C. Doubleday, J. Am. Chem. Soc. 95 (1973) 2735.

8. F. J. J. De Kanter and R. Kaptein, Chem. Phys. Lett. 62 (1979) 421.

9. K. L. Ivanov, K. Miesel, A. V. Yurkovskaya, S. E. Korchak, A. S. Kiryutin, and H.-M. Vieth, Appl. Magn. Reson. 30 (2006) 513.

10. K. L. Ivanov, A. V. Yurkovskaya, and H.-M. Vieth, J. Chem. Phys. 128 (2008) 154701.

11. K. Miesel, K. L. Ivanov, T. Koechling, A. V. Yurkovskaya, and H.-M. Vieth, Appl. Magn. Reson. 34 (2008) 423.

12. K. Miesel, K. L. Ivanov, A. V. Yurkovskaya, and H.-M. Vieth, Chem. Phys. Lett. 425 (2006) 71.

13. S. E. Korchak, K. L. Ivanov, A. V. Yurkovskaya, and H.-M. Vieth, Phys. Chem. Chem. Phys. 11 (2009) 11146.

14. E. B. Dücker, L. T. Kuhn, K. Münnemann, and C. Griesinger, J. Magn. Reson. 214 (2012) 159.

15. R. W. Adams, S. B. Duckett, R. A. Green, D. C. Williamson, and G. G. R. Green, J. Chem. Phys. 131 (2009) 194505.

16. K. D. Atkinson, M. J. Cowley, P. I. P. Elliott, S. B. Duckett, G. G. R. Green, J. Lopez-Serrano, and A. C. Whitwood, J. Am. Chem. Soc. 131 (2009) 13362.

17. L. Buljubasich, M. B. Franzoni, H. W. Spiess, and K. Münnemann, J. Magn. Reson. 219 (2012) 33.

18. S. Grosse, F. Gubaydullin, H. Scheelken, H.-M. Vieth, and A. V. Yurkovskaya, Appl. Magn. Reson. 17 (1999) 211.

19. S. Grosse, A. V. Yurkovskaya, J. Lopez, and H.-M. Vieth, J. Phys. Chem. A 105 (2001) 6311.

20. L. T. Kuhn, U. Bommerich, and J. Bargon, J. Phys. Chem. A 110 (2006) 3521.

21. S. Aime, W. Dastrù, R. Gobetto, and A. Viale, Org. Biomol. Chem. 3 (2005) 3948.

22. M. Roth, P. Kindervater, H.-P. Raich, J. Bargon, H.-W. Spiess, and K. Münnemann, Angew. Chemie Int. Ed. 49 (2010) 8358.

23. N. Amor, K. Hamilton, M. Küppers, U. Steinseifer, S. Appelt, B. Blümich, and T. SchmitzRode, ChemPhysChem 12 (2011) 2941.

24. D. Heller, S. Borns, W. Baumann, and R. Selke, Chem. Ber. 129 (1996) 85.

25. K. L. Ivanov, A. V. Yurkovskaya, and H.-M. Vieth, Z. Phys. Chem. 226 (2012) doi:10.1524/ zpch.2012.0269.

26. S. Schäublin, A. Höhener, and R. R. Ernst, J. Magn. Reson. 13 (1974) 196.

27. S. Schäublin, A. Wokaun, and R. R. Ernst, J. Magn. Reson. 27 (1977) 273.

28. K. L. Ivanov, K. Miesel, H.-M. Vieth, A. V. Yurkovskaya, and R. Z. Sagdeev, Z. Phys. Chem. 217 (2003) 1641.

29. J.-K. Vollenweider and H. Fischer, Chem. Phys. 108 (1986) 365.

30. H. Nies, H. Bauer, K. Roth, and D. Rewicki, J. Magn. Reson. 39 (1980) 521.

31. H. Jóhannesson, O. Axelsson, and M. Karlsson, C. R. Phys. 5 (2004) 315.

32. U. Bommerich, T. Trantzschel, S. Mulla-Osman, G. Buntkowsky, J. Bargon, and J. Bernarding, Phys. Chem. Chem. Phys. 12 (2010) 10309.

33. S. Aime, R. Gobetto, F. Reineri, and D. Canet, J. Magn. Reson. 178 (2006) 184.

34. S. E. Korchak, K. L. Ivanov, A. N. Pravdivtsev, A. V. Yurkovskaya, R. Kaptein, and H.-M. Vieth, J. Chem. Phys. 137 (2012) 094503.

35. M. Goldman and H. Jóhannesson, C. R. Phys. 6 (2005) 575.

36. M. Goldman, H. Jóhannesson, O. Axelsson, and M. Karlsson, C. R. Chim. 9 (2006) 357. 
37. E. Y. Chekmenev, V. A. Norton, D. P. Weitekamp, and P. Bhattacharya, J. Am. Chem. Soc. 131 (2009) 3164.

38. F. Reineri, S. Bouguet-Bonnet, and D. Canet, J. Magn. Reson. 210 (2011) 107.

39. M. Roth, A. Koch, P. Kindervater, J. Bargon, H. W. Spiess, and K. Münnemann, J. Magn. Reson. 204 (2010) 50.

40. M. Stephan, O. Kohlmann, H. G. Niessen, A. Eichhorn, and J. Bargon, Magn. Reson. Chem. 40 (2002) 157. 